\title{
Stability Analysis for a Discrete SIR Epidemic Model with Delay and General Nonlinear Incidence Function
}

\author{
Aboudramane Guiro*, Dramane Ouedraogo, Harouna Ouedraogo \\ Département de Mathématiques, UFR des Sciences et Techniques Université Nazi Boni de Bobo-Dioulasso \& LAboratoire de \\ Mathématique et d'Informatique (LAMI), Bobo-Dioulasso, Burkina Faso \\ Email: ^abouguiro@yahoo.fr, dramaneouedraogo268@yahoo.ca, oharounao@gmail.com
}

How to cite this paper: Guiro, A., Ouedraogo, D. and Ouedraogo, H. (2018) Stability Analysis for a Discrete SIR Epidemic Model with Delay and General Nonlinear Incidence Function. Applied Mathematics, 9, 1039-1054.

https://doi.org/10.4236/am.2018.99070

Received: July 16, 2018

Accepted: September 15, 2018

Published: September 18, 2018

Copyright ( $) 2018$ by authors and Scientific Research Publishing Inc. This work is licensed under the Creative Commons Attribution International License (CC BY 4.0).

http://creativecommons.org/licenses/by/4.0/

\begin{abstract}
In this paper, we construct a backward difference scheme for a class of $S I R$ epidemic model with general incidence $f$. The step size $\tau$ used in our discretization is one. The dynamical properties are investigated (positivity and the boundedness of solution). By constructing the Lyapunov function, the general incidence function $f$ must satisfy certain assumptions, under which, we establish the global stability of endemic equilibrium when $R_{0}>1$. The global stability of diseases-free equilibrium is also established when $R_{0} \leq 1$. In addition we present numerical results of the continuous and discrete model of the different class according to the value of basic reproduction number $R_{0}$.
\end{abstract}

\section{Keywords}

Discrete Model, Delay, Lyapunov Functional, Nonlinear Incidence, Backward Difference Scheme, Local Stability, Global Stability

\section{Introduction}

In certain epidemiological modeling, the population is generally divided into three classes which are susceptible represented by $\mathcal{S}$, infected individual represented by $I$ and recovered individual represented by $R$. This kind of mathematical model is noted SIR. Recently, many authors have studied the dynamical behavior of epidemic models (see [1] [2] [3] and references therein). There are two kinds of mathematical models: The continuous-time models described by differential equations, and the discrete-time models described by difference equations. The simplest forms of these models are Ordinary Differential Equations 
$(O D E s)$ [4] [5]. In [2], a discrete delay model is given to account for transmission by vectors (e.g. mosquitoes), where the delay $\tau$ is used to account for a latent period in the vector. Allowing the vectors latency periods to vary according to some distribution gives a model with a distributed delay [6].

The delay appears in the incidence term which is typically the only non-linearity, and is therefore the "cause" of all "interesting behavior". Various forms have been used for the incidence term, both for $O D E s$ and for delay equations. Common forms include mass action $\beta S I$ [6] [7] [8], saturating incidence $\beta S \frac{I}{1+c I}$ [9] [10], and standard (or proportional) incidence $\beta \frac{S I}{N}$

[4] $(N=S+I)$. Changing the form of the incidence function can potentially change the behavior of the system.

In this paper we study the discrete mathematical model which result from the continuous-time model presented and study in [11] by C. Connell McCluskey. From this we use the general incidence term $\beta \sum_{j=0}^{h} k(j) f\left(S_{n}, I_{n}^{j}\right)$, where $h>0$ is a time delay. We choose the constant $\beta$ so that $\sum_{j=0}^{h} k(j)=1$. The discrete model is obtained by using the backward Euler method.

The studied of discrete epidemic models is motivate by the fact that there occur situations such that constructing discrete epidemic models is more appropriate approach to understand disease transmission dynamics and to evaluate eradication policies because they permit arbitrary time-step units, preserving the basic features of corresponding continuous-time models [12]. Furthermore, this allows better use of statistical data for numerical simulations due to the reason that the infection data are compiled at discrete given time intervals. For a discrete epidemic model with immigration of infectives, Jang and Elaydi [13] showed the global asymptotic stability of the disease-free equilibrium, the local asymptotic stability of the endemic equilibrium and the strong persistence of susceptible class by means of the nonstandard discretization method. In he's recent work, using a discretization called " mixed type" formula in Izzo and Vecchio [14] and Izzo et al. [15], Sekiguchi [16] obtained the permanence of a class of SIR discrete epidemic models with one delay and SEIRS (Susceptible-Latent-Infected-Recovered-Susceptible) discrete epidemic model with two delay if an endemic equilibrium of each model exists.

This paper is organized as follows. In Section 2, we give the discrete model, the equilibrium point and the reproduction rate $R_{0}$. In Section 3, the positivity and boundedness of the solution of system (3) are obtained. In addition we proved the existence and uniqueness of disease-free equilibrium $E_{0}$ and endemic equilibrium $E^{*}$. In Section 4 , we study the stability of disease-free equilibrium point for $R_{0} \leq 1$. In Section 5 , we study the global stability of the endemic equilibrium point for $R_{0}>1$. In section 6 , we give the numerical result and their comment. In the last part we give the conclusion. 


\section{Discrete Mathematical Model}

In this section we describe the discrete mathematical model derived by the continuous time model study in [11], by C. Connell McCluskey. This continuous time model is given by:

$$
\left\{\begin{array}{l}
\dot{S}=B-\mu_{S} S-\beta \int_{0}^{h} k(\tau) f\left(S, I_{\tau}\right) \mathrm{d} \tau, \\
\dot{I}=\beta \int_{0}^{h} k(\tau) f\left(S, I_{\tau}\right) \mathrm{d} \tau-\left(\mu_{I}+\gamma\right) I, \\
\dot{R}=\gamma I-\mu_{R} R
\end{array}\right.
$$

where $I_{\tau}=I(t-\tau)$.

1) A population is divided into susceptible, infectious and recovered classes with sizes $S=S(t), I=I(t)$ and $R=R(t)$ respectively.

2) $B$ is the recruitment of new individuals, it is into the susceptible class.

3) $\mu_{S}, \mu_{I}$ and $\mu_{R}$ denote respectively the death rates of susceptible, infectious and recovered class.

4) The total exit rate for infectious is $\mu_{I}+\gamma$, which, for biological reasons we assume is at least as large as $\mu_{S}$; that is, $\mu_{I}+\gamma \geq \mu_{S}$.

5) The incidence at time $t$ is $\beta \int_{0}^{h} k(\tau) f\left(S, I_{\tau}\right) \mathrm{d} \tau$ where the maximum delay $h>0, k$ is a Lebesgue integral function which gives the relative infectivity of vectors of different infection ages. We choose $\beta$ so that $\int_{0}^{h} k(\tau) \mathrm{d} \tau=1$. It is assumed that the support of $k$ has positive measure in any open interval having supremum $h$ so the interval of integration is not artificially extended by concluding with an interval for which the integral is automatically zero.

The form of the function $f$ is of fundamental importance. In this paper we use a general incidence function used in one of he's discrete version. So we use assumption:

H1 $f$ is a non-negative differentiable function on the non-negative quadrant. Furthermore, $f$ is positive if and only if both arguments are positive.

$\mathrm{H} 2$ for all $(S, I) \in \mathbb{R}_{+}^{2} f(S, 0)=f(0, I)=0$.

The partial derivative of $f$ are denoted by $f_{1}$ and $f_{2}$ from the first and second variable.

H3 $f\left(S_{n}, I_{n}^{j}\right) \leq f_{2}\left(S^{0}, 0\right) I_{n}$ for all $n$.

H4 $\frac{f\left(S^{*}, I^{*}\right)}{f\left(S_{n+1}, I_{n+1}^{j}\right)} \leq \frac{S^{*}}{S_{n+1}} \leq \frac{I^{*}}{I_{n+1}}$ for all $n$.

Now, we use the backward Euler difference scheme to discretize the model (1). The time step size of our discretization is one. Thus, we obtain the following discrete $S I R$ epidemic model with nonlinear general incidence given by:

$$
\left\{\begin{array}{l}
S_{n+1}-S_{n}=B-\mu_{S} S_{n+1}-\beta \sum_{j=0}^{h} k(j) f\left(S_{n+1}, I_{n+1}^{j}\right) \\
I_{n+1}-I_{n}=\beta \sum_{j=0}^{h} k(j) f\left(S_{n+1}, I_{n+1}^{j}\right)-\left(\mu_{I}+\gamma\right) I_{n+1} \\
R_{n+1}-R_{n}=\gamma I_{n+1}-\mu_{R} R_{n+1}
\end{array}\right.
$$


where $I^{j}=I(t-j)$.

Since $R$ does not appear in the first and second equations of system above, it is sufficient to analyses the behavior of solutions of the following system:

$$
\left\{\begin{array}{l}
S_{n+1}-S_{n}=B-\mu_{S} S_{n+1}-\beta \sum_{j=0}^{h} k(j) f\left(S_{n+1}, I_{n+1}^{j}\right) \\
I_{n+1}-I_{n}=\beta \sum_{j=0}^{h} k(j) f\left(S_{n+1}, I_{n+1}^{j}\right)-\left(\mu_{I}+\gamma\right) I_{n+1}
\end{array}\right.
$$

The constants $B, \mu_{S}, \mu_{I}, \gamma$ and the relation between this constants are given above. In the discrete model the incidence function at time $t$ is

$$
\beta \sum_{j=0}^{h} k(j) f\left(S_{n+1}, I_{n+1}^{j}\right),
$$

where the maximum delay $h>0$. Let $E(S, I)$ be a equilibrium point model of (3) so we have,

$$
\left\{\begin{array}{l}
B-\mu_{S} S-\beta f(S, I)=0 \\
\beta f(S, I)-\left(\mu_{I}+\gamma\right) I=0
\end{array}\right.
$$

By adding the equations of system above we get

$$
\begin{aligned}
& B-\mu_{S} S-\left(\mu_{I}+\gamma\right) I=0 \\
& \Rightarrow S=\frac{B-\left(\mu_{I}+\gamma\right) I}{\mu_{S}} .
\end{aligned}
$$

Let $E_{0}$ and $E^{*}$ be respectively disease-free equilibrium and endemic equilibrium point of model (3). The disease-free equilibrium correspond to the case where the infectious class is nil $(I=0)$. Thus, we have $E_{0}=\left(S_{0}, 0\right)$; with $S_{0}=\frac{B}{\mu_{S}}$. The endemic equilibrium $E^{*}$ is given by: $E^{*}=\left(S^{*}, I^{*}\right)$; with

$$
S^{*}=\frac{B-\left(\mu_{I}+\gamma\right) I^{*}}{\mu_{S}}
$$

Proposition 2.1. The basic reproduction number is given by $R_{0}=\frac{\beta f_{2}\left(E_{0}\right)}{\mu_{I}+\gamma}$.

Proof: The Jacobian matrix of system (3) at equilibrium $E_{0}$ is define by:

$$
J_{E_{0}}=\left(\begin{array}{cc}
-\mu_{S}-\beta f_{1}\left(E_{0}\right) & -\beta f_{2}\left(E_{0}\right) \\
\beta f_{1}\left(E_{0}\right) & \beta f_{2}\left(E_{0}\right)-\left(\mu_{I}+\gamma\right)
\end{array}\right) .
$$

Let

$$
\begin{gathered}
A=\beta_{2}\left(E_{0}\right)-\left(\mu_{I}+\gamma\right), \\
M=\beta f_{2}\left(E_{0}\right) \text { et } D=\mu_{I}+\gamma .
\end{gathered}
$$

Thus, we have:

$$
\begin{gathered}
R_{0}=M D^{-1} \\
R_{0}=\frac{\beta f_{2}\left(E_{0}\right)}{\mu_{I}+\gamma} .
\end{gathered}
$$




\section{Basic Properties}

We suppose that initial condition of system (3) satisfy:

$$
S(0)>0 \text { and } I(\theta)=\Phi(\theta) \text { for all } \theta \in[-h, 0],
$$

where $\Phi \in \mathcal{C}=C\left([-h, 0], \mathbb{R}_{+}\right)$, the space of continuous functions from $[-h, 0]$ to $\mathbb{R}_{+}$equipped with the sup norm: $\|\Phi\|=\sup _{\theta \in[-h, 0]} \Phi(\theta)$. Standard theory of functional differential equations [17] can be used to show that the solution of (1) exist and are differentiable for all $t>0$. We assume any initial condition for which the disease is initially present satisfies $I(\theta)>0$ for all $\theta \in[-h, 0]$.

Lemma 3.1. Let $\left(S_{n}, I_{n}\right)$ be a solution of system (3) with initial condition (11), then we have $S_{n}>0$ and $I_{n}>0$ for all $n$.

Proof: Assume that $S_{n}>0$ and $I_{n}>0$. From system (3) we have the following system:

$$
\left\{\begin{array}{l}
\left(1+\mu_{S}\right) S_{n+1}=B+S_{n}-\beta \sum_{j=0}^{h} k(j) f\left(S_{n+1}, I_{n+1}^{j}\right) \\
\left(1+\mu_{I}+\gamma\right) I_{n+1}=I_{n}+\beta \sum_{j=0}^{h} k(j) f\left(S_{n+1}, I_{n+1}^{j}\right) .
\end{array}\right.
$$

By using second equation of system above and the fact that $I_{n}>0$, we have, $I_{n+1}>0$. So $I_{n}>0, \forall n \in \mathbb{N}$. From the non-negativity of $S_{n+1}$ we used the assumption H4. Thus, $S_{n}, I_{n}>0$ for all $n$.

Lemma 3.2. Any solution $\left(S_{n}, I_{n}\right)$ of system (3), with initial condition (11) satisfy

$$
\lim \sup _{n \rightarrow+\infty}\left(S_{n}+I_{n}\right) \leq \frac{B}{\mu_{S}} .
$$

Proof: Let $N_{n}=S_{n}+I_{n}$ and $N_{n+1}=S_{n+1}+I_{n+1}$; for biological reasons we assume is at least as large as $\mu_{S}$; that is, $\mu_{I}+\gamma \geq \mu_{S}$.

By adding the different equation of system (3) we get:

$$
\begin{gathered}
N_{n+1}-N_{n}=B-\mu_{S} S_{n+1}-\left(\mu_{I}+\gamma\right) I_{n+1} \\
\leq B-\mu_{S} S_{n+1}-\mu_{S} I_{n+1} \\
\leq B-\mu_{S}\left(S_{n+1}+I_{n+1}\right) \\
\leq B-\mu_{S} N_{n+1} \\
\mu_{S} N_{n+1}+N_{n+1}-N_{n} \leq B \\
\limsup \mu_{S} N_{n+1}+N_{n+1}-N_{n}=\limsup \mu_{n \rightarrow+\infty} N_{n+1} \\
\Rightarrow \lim \sup _{n \rightarrow+\infty} \mu_{S} N_{n+1} \leq B \\
\Rightarrow \lim _{n \rightarrow+\infty} \sup _{n+1} \leq \frac{B}{\mu_{S}} .
\end{gathered}
$$

Hence, we have

$$
\lim \sup _{n \rightarrow+\infty}\left(S_{n}+I_{n}\right) \leq \frac{B}{\mu_{S}} .
$$

Proposition 3.1. 
1) When $R_{0}<1$, then model (3) has only a unique disease-free equilibrium $E_{0}=\left(S^{0}, 0\right)$.

2) When $R_{0}>1$, then model (3) has only a unique endemic equilibrium $E^{*}=\left(S^{*}, I^{*}\right)$.

Proof: Any equilibrium point $E=(S, I)$ of system (3) verified the following system:

$$
\left\{\begin{array}{l}
B-\mu_{S} S-\beta f(S, I)=0 \\
\beta f(S, I)-\left(\mu_{I}+\gamma\right) I=0 .
\end{array}\right.
$$

By using the second equation of system above we have:

$$
\beta \frac{f(S, I)}{I}=\left(\mu_{I}+\gamma\right) \text {. }
$$

So we can consider the function $G$ defined by,

$$
G(I)=\beta \frac{f\left(S^{0}-\frac{\mu_{I}+\gamma}{\mu_{S}} I, I\right)}{I}-\left(\mu_{I}+\gamma\right) .
$$

Hence we have

$$
\begin{aligned}
\lim _{I \rightarrow 0^{+}} G(I) & =\frac{\beta \partial f}{\partial I}\left(S^{0}, 0\right)-\left(\mu_{I}+\gamma\right) \\
& =\left(\mu_{I}+\gamma\right)\left(\frac{\beta f_{2}\left(S^{0}, 0\right)}{\mu_{I}+\gamma}-1\right) \\
& =\left(\mu_{I}+\gamma\right)\left(R_{0}-1\right) .
\end{aligned}
$$

And also we have,

$$
G(\bar{I})=-\left(\mu_{I}+\gamma\right) \text {, where } \bar{I}=\frac{\mu_{S} S^{0}}{\mu_{I}+\gamma} .
$$

when $R_{0} \leq 1$, we have $\lim _{I \rightarrow 0^{+}} G(I) \leq 0$. Consequently, there is not any $I^{*}>0$ such that $G\left(I^{*}\right)=0$. Therefore, model (3) has a unique disease-free equilibrium $E_{0}$.

When, $R_{0}>1$, we have $\lim _{I \rightarrow 0^{+}} G(I)>0$. Therefore, there exists a unique $\left.I^{*} \in\right] 0 ; \bar{I}\left[\right.$ such that $G\left(I^{*}\right)=0$.

This implies that model (3) has unique endemic equilibrium $E^{*}=\left(S^{*}, I^{*}\right)$.

Remark 3.1. The space $K=\mathbb{R}_{+} \times \mathcal{C}$ is positively invariant and attracting domain for system (3).

Now, let us analyze the behavior of system (3) when the basic reproduction rate $R_{0}$ is less than one.

\section{Stability of the Disease-Free Equilibrium}

In this section, we study the stability of diseases-free equilibrium $E_{0}=\left(S^{0}, 0\right)$, with $S^{0}=\frac{B}{\mu_{S}}$. 
Theorem 4.1. If $R_{0} \leq 1$, then the diseases-free equilibrium $E_{0}$ of system(3) is locally asymptotically stable.

Proof: The linearization of system (3) at diseases-free equilibrium point $E_{0}$ is given by:

$$
\left\{\begin{array}{l}
S_{n+1}-S_{n}=\left[-\mu_{S}-\beta f_{1}\left(E_{0}\right)\right] S_{n+1}-\beta f_{2}\left(E_{0}\right) I_{n+1} \\
I_{n+1}-I_{n}=\beta f_{1}\left(E_{0}\right) S_{n+1}+\left[\beta f_{2}\left(E_{0}\right)-\left(\mu_{I}+\gamma\right)\right] I_{n+1} .
\end{array}\right.
$$

Thus, we have:

$$
\left\{\begin{array}{l}
{\left[1+\mu_{S}+\beta f_{1}\left(E_{0}\right)\right] S_{n+1}+\beta f_{2}\left(E_{0}\right) I_{n+1}=S_{n}} \\
-\beta f_{1}\left(E_{0}\right) S_{n+1}+\left[1-\beta f_{2}\left(E_{0}\right)+\left(\mu_{I}+\gamma\right)\right] I_{n+1}=I_{n} .
\end{array}\right.
$$

The matrix $M$ associate of the linearization (23) is given by:

$$
M=\left(\begin{array}{cc}
1+\mu_{S}+\beta f_{1}\left(E_{0}\right) & \beta f_{2}\left(E_{0}\right) \\
-\beta f_{1}\left(E_{0}\right) & 1-\beta f_{2}\left(E_{0}\right)+\left(\mu_{I}+\gamma\right)
\end{array}\right),
$$

and the linearization system can be rewrite by:

$$
X_{n+1}=M^{-1} X_{n},
$$

with $X_{n}=\left(S_{n}, I_{n}\right)^{t}$.

The model (3) is locally asymptotically stable at diseases-free equilibrium point $E_{0}$ if all eigenvalue of matrix $M$ is greater than one.

Let $P(X)$ be characteristic polynomial associate of matrix $M$. We have,

$$
\begin{aligned}
P(X)= & \operatorname{det}\left(M-X I_{2}\right) \\
= & \left(1+\mu_{S}+\beta f_{1}\left(E_{0}\right)-X\right)\left(1-\beta f_{2}\left(E_{0}\right)+\left(\mu_{I}+\gamma\right)-X\right) \\
& +\left(\beta f_{1}\left(E_{0}\right)\right)\left(\beta f_{2}\left(E_{0}\right)\right) .
\end{aligned}
$$

Let $\lambda$ be a eigenvalue of matrix $M$, thus $P(\lambda)=0$. This implies that:

$\left(1+\mu_{S}+\beta f_{1}\left(E_{0}\right)-\lambda\right)\left(1-\beta f_{2}\left(E_{0}\right)+\left(\mu_{I}+\gamma\right)-\lambda\right)+\left(\beta f_{1}\left(E_{0}\right)\right)\left(\beta f_{2}\left(E_{0}\right)\right)=0$.

From the Equation (27) we have

$$
\left(1+\mu_{S}+\beta f_{1}\left(E_{0}\right)-\lambda\right)\left(1-\beta f_{2}\left(E_{0}\right)+\left(\mu_{I}+\gamma\right)-\lambda\right)=-\left(\beta f_{1}\left(E_{0}\right)\right)\left(\beta f_{2}\left(E_{0}\right)\right) .
$$

By using the second member of (28), the fact that $R_{0}<1$ and we assume that the matrix $M$ have a eigenvalue which is less than one. So we have:

$$
\begin{aligned}
& -\left(\beta f_{1}\left(E_{0}\right)\right)\left(\beta f_{2}\left(E_{0}\right)\right)=\left(\beta f_{1}\left(E_{0}\right)\right)\left(-\beta f_{2}\left(E_{0}\right)\right) \\
& \leq\left(\mu_{S}+\beta f_{1}\left(E_{0}\right)\right)\left(-\beta f_{2}\left(E_{0}\right)+\left(\mu_{I}+\gamma\right)\right) \\
& <\left(1+\mu_{S}+\beta f_{1}\left(E_{0}\right)-\lambda\right)\left(1-\beta f_{2}\left(E_{0}\right)+\left(\mu_{I}+\gamma\right)-\lambda\right)
\end{aligned}
$$

as a result of, the Equation (28) cannot have roots which is less than one. Hence, $E_{0}$ is locally asymptotically stable according to the theorem 2 in [18].

Theorem 4.2. When $R_{0} \leq 1$, the disease-free equilibrium $E_{0}$ of system (3) is globally asymptotically stable in $K$.

Proof: In this proof we used the comparison theorem [19]. By using the second equation of system (3) and the assumption $\mathrm{H} 3$; we get: 


$$
\begin{aligned}
I_{n} & =\left(1+\left(\mu_{I}+\gamma\right)\right) I_{n+1}-\beta \sum_{j=0}^{h} k(j) f\left(S_{n+1}, I_{n+1}^{j}\right) \\
& \geq\left(1+\left(\mu_{I}+\gamma\right)-\beta \sum_{j=0}^{h} k(j) f\left(S^{0}, 0\right)\right) I_{n+1} .
\end{aligned}
$$

Hence we have:

$$
I_{n} \geq\left(1+\left(\mu_{I}+\gamma\right)-\beta f_{2}\left(S^{0}, 0\right)\right) I_{n+1}
$$

Thus,

$$
M^{-1} I_{n} \geq I_{n+1} \text { with } M=1+\left(\mu_{I}+\gamma\right)-\beta f_{2}\left(S^{0}, 0\right) .
$$

By using the fact $R_{0} \leq 1$ we have $\mu_{I}+\gamma-\beta f_{2}\left(S^{0}, 0\right) \geq 0$. So the constant $M$ is greater than one. we conclude that the linearized Equation (32) is stable whenever $R_{0} \leq 1$. By a standard comparison theorem [19], $I_{n} \rightarrow 0$ as $n \rightarrow+\infty$ for Equation (32) and substituting $I_{n}=0$ in system (3) we get $S_{n} \rightarrow S^{0}, I_{n} \rightarrow 0$ as $n \rightarrow+\infty$. Thus, $\left(S_{n}, I_{n}\right) \rightarrow\left(S^{0}, 0\right)$ as $n \rightarrow+\infty$ for system (3), when $R_{0} \leq 1$. Therefore $E_{0}$ is globally asymptotically stable in the positively invariant set $K$ if $R_{0} \leq 1$.

\section{Global Stability of the Endemic Equilibrium}

In this section, we study the stability the stability of endemic equilibrium $E^{*}=\left(S^{*}, I^{*}\right)$, with

$$
S^{*}=S^{0}-\frac{\mu_{I}+\gamma}{\mu_{S}} I^{*} .
$$

Theorem 5.1. If $R_{0}>1$, then the endemic equilibrium $E^{*}$ of system (3) is globally asymptotically stable.

Proof: From the equation of system (3), at endemic equilibrium $E^{*}$, we have:

$$
B=\mu_{S} S^{*}+\beta \sum_{j=0}^{h} k(j) f\left(S^{*}, I^{*}\right)
$$

and

$$
\left(\mu_{I}+\gamma\right) I^{*}=\beta f\left(S^{*}, I^{*}\right),
$$

which will be used as substitutions in the calculations below. Let $g(x)=x-1-\ln x$ and

$$
\begin{gathered}
V_{S}(n)=S^{*} g\left(\frac{S_{n}}{S^{*}}\right) \\
V_{I}(n)=I^{*} g\left(\frac{I_{n}}{I^{*}}\right) \\
V_{+}(n)=\sum_{j=0}^{h} \alpha(j) g\left(\frac{I_{n-j}}{I^{*}}\right),
\end{gathered}
$$

where 


$$
\alpha(j)=\beta \sum_{s=j}^{h} k(s) f\left(S^{*}, I^{*}\right) .
$$

We will study the behavior of the Lyapunov functional

$$
V(n)=V_{S}(n)+V_{I}(n)+V_{+}(n) ;
$$

which satisfies $V_{n} \geq 0$ with equality if and only if

$$
\frac{S_{n}}{S^{*}}=\frac{I_{n}}{I^{*}}=1 \text { and } \frac{I_{n-j}}{I^{*}}=1
$$

for all $j \in[0, h]$. For clarity, the difference $V_{S}(n+1)-V_{S}(n), V_{I}(n+1)-V_{I}(n)$ and $V_{+}(n+1)-V_{+}(n)$ will be calculated separately and then combined to obtain $V(n+1)-V(n)$.

Calculation of the variation $V_{S}(n+1)-V_{S}(n)$ : in this calculation, we used the value theorem and we assume that $S_{n+1}>S_{n}$. Note that we have the same result when $S_{n}>S_{n+1}$.

$$
\begin{aligned}
& V_{S}(n+1)-V_{S}(n)=S^{*} g\left(\frac{S_{n+1}}{S^{*}}\right)-S^{*} g\left(\frac{S_{n}}{S^{*}}\right) \\
& =\left(1-S^{*} \frac{\ln S_{n+1}-\ln S_{n}}{S_{n+1}-S_{n}}\right)\left(S_{n+1}-S_{n}\right) \leq\left(1-\frac{S^{*}}{S_{n+1}}\right)\left(S_{n+1}-S_{n}\right) \\
& \leq\left(1-\frac{S^{*}}{S_{n+1}}\right)\left(B-\mu_{S} S_{n+1}-\beta \sum_{j=0}^{h} k(j) f\left(S_{n+1}, I_{n+1}^{j}\right)\right) \\
& \leq\left(1-\frac{S^{*}}{S_{n+1}}\right)\left(\mu_{S} S^{*}+\beta f\left(S^{*}, I^{*}\right) \sum_{j=0}^{h} k(j)-\mu_{S} S_{n+1}-\beta \sum_{j=0}^{h} k(j) f\left(S_{n+1}, I_{n+1}^{j}\right)\right) \\
& \leq-\mu_{S} \frac{\left(S_{n+1}-S^{*}\right)^{2}}{S_{n+1}}+\beta \sum_{j=0}^{h} k(j) f\left(S^{*}, I^{*}\right)\left[\left(1-\frac{S^{*}}{S_{n+1}}\right)\left(1-\frac{f\left(S_{n+1}, I_{n+1}^{j}\right)}{f\left(S^{*}, I^{*}\right)}\right)\right] \\
& \leq-\mu_{S} \frac{\left(S_{n+1}-S^{*}\right)^{2}}{S_{n+1}}+\beta \sum_{j=0}^{h} k(j) f\left(S^{*}, I^{*}\right)\left(1-\frac{f\left(S_{n+1}, I_{n+1}^{j}\right)}{f\left(S^{*}, I^{*}\right)}\right. \\
& \left.-\frac{S^{*}}{S_{n+1}}+\frac{S^{*}}{S_{n+1}} \frac{f\left(S_{n+1}, I_{n+1}^{j}\right)}{f\left(S^{*}, I^{*}\right)}\right) \\
& V_{S}(n+1)-V_{S}(n) \\
& \quad=-\mu_{S} \frac{\left(S_{n+1}-S^{*}\right)^{2}}{S_{n+1}}+\beta \sum_{j=0}^{h} k(j) f\left(S^{*}, I^{*}\right)\left(1-\frac{f\left(S_{n+1}, I_{n+1}^{j}\right)}{f\left(S^{*}, I^{*}\right)}\right. \\
& \left.\quad-\frac{S^{*}}{S_{n+1}}+\frac{S^{*}}{S_{n+1}} \frac{f\left(S_{n+1}, I_{n+1}^{j}\right)}{f\left(S^{*}, I^{*}\right)}\right)
\end{aligned}
$$

Let us calculate of the variation $V_{I}(n+1)-V_{I}(n)$ : in this calculation we used the mane value theorem and we assume that $I_{n+1}>I_{n}$. Note that we have the same result when $I_{n}>I_{n+1}$.

$$
V_{I}(n+1)-V_{I}(n)=I^{*} g\left(\frac{I_{n+1}}{I^{*}}\right)-I^{*} g\left(\frac{I_{n}}{I^{*}}\right)
$$




$$
\begin{aligned}
& =\left(1-I^{*} \frac{\ln I_{n+1}-\ln I_{n}}{I_{n+1}-I_{n}}\right)\left(I_{n+1}-I_{n}\right) \\
& =\left(1-I^{*} \frac{\ln I_{n+1}-\ln I_{n}}{I_{n+1}-I_{n}}\right)\left(\beta \sum_{j=0}^{h} k(j) f\left(S_{n+1}, I_{n+1}^{j}\right)-\left(\mu_{I}+\gamma\right) I_{n+1}\right) \\
& \leq\left(1-\frac{I^{*}}{I_{n+1}}\right)\left(\beta \sum_{j=0}^{h} k(j) f\left(S_{n+1}, I_{n+1}^{j}\right)-\left(\mu_{I}+\gamma\right) I^{*} \frac{I_{n+1}}{I^{*}}\right) \\
& \leq\left(1-\frac{I^{*}}{I_{n+1}}\right)\left(\beta \sum_{j=0}^{h} k(j) f\left(S_{n+1}, I_{n+1}^{j}\right)-\beta f\left(S^{*}, I^{*}\right) \sum_{j=0}^{h} k(j) \frac{I_{n+1}}{I^{*}}\right) \\
& \leq\left(1-\frac{I^{*}}{I_{n+1}}\right)\left(\beta \sum_{j=0}^{h} k(j)\left[f\left(S_{n+1}, I_{n+1}^{j}\right)-f\left(S^{*}, I^{*}\right) \frac{I_{n+1}}{I^{*}}\right]\right) \\
& \leq \beta \sum_{j=0}^{h} k(j) f\left(S^{*}, I^{*}\right)\left[\left(1-\frac{I^{*}}{I_{n+1}}\right)\left(\frac{f\left(S_{n+1}, I_{n+1}^{j}\right)}{f\left(S^{*}, I^{*}\right)}-\frac{\left.\left.I_{n+1}\right)\right]}{I^{*}}\right)\right] \\
& \leq \beta \sum_{j=0}^{h} k(j) f\left(S^{*}, I^{*}\right)\left[\frac{f\left(S_{n+1}, I_{n+1}^{j}\right)}{f\left(S^{*}, I^{*}\right)}-\frac{I_{n+1}}{I^{*}}-\frac{I^{*}}{I_{n+1}} \frac{f\left(S_{n+1}, I_{n+1}^{j}\right)}{f\left(S^{*}, I^{*}\right)}+1\right], \\
& V_{I}(n+1)-V_{I}(n) \\
& \leq \beta f\left(S^{*}, I^{*}\right) \sum_{j=0}^{h} k(j)\left[\frac{f\left(S_{n+1}, I_{n+1}^{j}\right)}{f\left(S^{*}, I^{*}\right)}-\frac{I_{n+1}}{I^{*}}-\frac{I^{*}}{I_{n+1}} \frac{f\left(S_{n+1}, I_{n+1}^{j}\right)}{f\left(S^{*}, I^{*}\right)}+1\right] .
\end{aligned}
$$

Let now evaluate the variation $V_{+}(n+1)-V_{+}(n)$ :

$$
\begin{aligned}
V_{+}(n+1)-V_{+}(n) & =\sum_{j=0}^{h} \alpha(j) g\left(\frac{I_{n+1-j}}{I^{*}}\right)-\sum_{j=0}^{h} \alpha(j) g\left(\frac{I_{n-j}}{I^{*}}\right) \\
& =\alpha(0) g\left(\frac{I_{n+1}}{I^{*}}\right)-\alpha(0) g\left(\frac{I_{n-j}}{I^{*}}\right) \\
& =\sum_{j=0}^{h} \beta k(j) f\left(S^{*}, I^{*}\right)\left[g\left(\frac{I_{n+1}}{I^{*}}\right)-g\left(\frac{I_{n-j}}{I^{*}}\right)\right] \\
& =\sum_{j=0}^{h} \beta k(j) f\left(S^{*}, I^{*}\right)\left[\frac{I_{n+1}}{I^{*}}-\frac{I_{n-j}}{I^{*}}-\ln \frac{I_{n+1}}{I^{*}}+\ln \frac{I_{n-j}}{I^{*}}\right] \\
V_{+}(n+1)-V_{+}(n) & =\beta \sum_{j=0}^{h} k(j) f\left(S^{*}, I^{*}\right)\left[\frac{I_{n+1}}{I^{*}}-\frac{I_{n-j}}{I^{*}}-\ln \frac{I_{n+1}}{I^{*}}+\ln \frac{I_{n-j}}{I^{*}}\right] .
\end{aligned}
$$

By adding Equations (42)-(44) we obtain

$$
\begin{aligned}
& V(n+1)-V(n) \\
& \leq-\mu_{S} \frac{\left(S_{n+1}-S^{*}\right)^{2}}{S_{n+1}} \\
& +\beta \sum_{j=0}^{h} k(j) f\left(S^{*}, I^{*}\right)\left(1-\frac{f\left(S_{n+1}, I_{n+1}^{j}\right)}{f\left(S^{*}, I^{*}\right)}-\frac{S^{*}}{S_{n+1}}+\frac{S^{*}}{S_{n+1}} \frac{f\left(S_{n+1}, I_{n+1}^{j}\right)}{f\left(S^{*}, I^{*}\right)}\right) \\
& +\beta \sum_{j=0}^{h} k(j) f\left(S^{*}, I^{*}\right)\left[\frac{f\left(S_{n+1}, I_{n+1}^{j}\right)}{f\left(S^{*}, I^{*}\right)}-\frac{I_{n+1}}{I^{*}}-\frac{I^{*}}{I_{n+1}} \frac{f\left(S_{n+1}, I_{n+1}^{j}\right)}{f\left(S^{*}, I^{*}\right)}+1\right]
\end{aligned}
$$




$$
\begin{aligned}
& +\beta \sum_{j=0}^{h} k(j) f\left(S^{*}, I^{*}\right)\left[\frac{I_{n+1}}{I^{*}}-\frac{I_{n-j}}{I^{*}}-\ln \frac{I_{n+1}}{I^{*}}+\ln \frac{I_{n-j}}{I^{*}}\right] \\
\leq & -\mu_{S} \frac{\left(S_{n+1}-S^{*}\right)^{2}}{S_{n+1}}+\beta \sum_{j=0}^{h} k(j) f\left(S^{*}, I^{*}\right) Q(j),
\end{aligned}
$$

where

$Q(j)=2-\frac{S^{*}}{S_{n+1}}+\frac{S^{*}}{S_{n+1}} \frac{f\left(S_{n+1}, I_{n+1}^{j}\right)}{f\left(S^{*}, I^{*}\right)}-\frac{I^{*}}{I_{n+1}} \frac{f\left(S_{n+1}, I_{n+1}^{j}\right)}{f\left(S^{*}, I^{*}\right)}-\frac{I_{n-j}}{I^{*}}-\ln \frac{I_{n+1}}{I^{*}}+\ln \frac{I_{n-j}}{I^{*}}$.

By adding and subtracting $1+\ln \frac{S^{*}}{S_{n+1}} \frac{f\left(S_{n+1}, I_{n+1}^{j}\right)}{f\left(S^{*}, I^{*}\right)}+\ln \frac{I^{*}}{I_{n+1}} \frac{f\left(S_{n+1}, I_{n+1}^{j}\right)}{f\left(S^{*}, I^{*}\right)}$ to

we obtain:

$$
\begin{aligned}
Q(j)= & \left.-\frac{I_{n-j}}{I^{*}}+1+\ln \frac{I_{n-j}}{I^{*}}\right)+1-\frac{I^{*}}{I_{n+1}} \frac{f\left(S_{n+1}, I_{n+1}^{j}\right)}{f\left(S^{*}, I^{*}\right)} \\
& -\ln \frac{I_{n+1}}{I^{*}}-\frac{S^{*}}{S_{n+1}}+\frac{S^{*}}{S_{n+1}} \frac{f\left(S_{n+1}, I_{n+1}^{j}\right)}{f\left(S^{*}, I^{*}\right)} \\
= & -g\left(\frac{I_{n-j}}{I^{*}}\right)+\left(-\frac{I^{*}}{I_{n+1}} \frac{f\left(S_{n+1}, I_{n+1}^{j}\right)}{f\left(S^{*}, I^{*}\right)}+1+\ln \frac{I^{*}}{I_{n+1}} \frac{f\left(S_{n+1}, I_{n+1}^{j}\right)}{f\left(S^{*}, I^{*}\right)}\right) \\
& +\left(-\frac{S^{*}}{S_{n+1}}+1+\ln \frac{S^{*}}{S_{n+1}}\right)+\left(\frac{S^{*}}{S_{n+1}} \frac{f\left(S_{n+1}, I_{n+1}^{j}\right)}{f\left(S^{*}, I^{*}\right)}-1-\ln \frac{S^{*}}{S_{n+1}} \frac{f\left(S_{n+1}, I_{n+1}^{j}\right)}{f\left(S^{*}, I^{*}\right)}\right) \\
Q(j)= & -g\left(\frac{I_{n-j}}{I^{*}}\right)-g\left(\frac{I^{*}}{I_{n+1}} \frac{f\left(S_{n+1}, I_{n+1}^{j}\right)}{f\left(S^{*}, I^{*}\right)}\right)-g\left(\frac{S^{*}}{S_{n+1}}\right)+g\left(\frac{S^{*}}{S_{n+1}} \frac{f\left(S_{n+1}, I_{n+1}^{j}\right)}{f\left(S^{*}, I^{*}\right)}\right) .(
\end{aligned}
$$

By using the assumption $\mathrm{H} 4$ and the fact that the function $g$ is increasing on ] $1,+\infty[$, we have

$$
g\left(\frac{S^{*}}{S_{n+1}} \frac{f\left(S_{n+1}, I_{n+1}^{j}\right)}{f\left(S^{*}, I^{*}\right)}\right)-g\left(\frac{I^{*}}{I_{n+1}} \frac{f\left(S_{n+1}, I_{n+1}^{j}\right)}{f\left(S^{*}, I^{*}\right)}\right) \leq 0
$$

so we have $Q(j) \leq 0$. This implies that $V(n+1)-V(n) \leq 0$. Hence, by the Lyapunovs theorems on the global asymptotical stability for difference equations [20], we obtain that the endemic equilibrium $E^{*}$ is globally asymptotically stable.

\section{Simulation and Comments}

In this section, we presented a numerical result of continuous-time model (1) and the discrete one (2) study above. From this we used a particular incidence function define by $f(S, I)=\frac{\beta S I_{\tau}}{1+c I_{\tau}}$, which is the saturating incidence. In addition we discuss from the different value of the basic reproduction number $R_{0}$. We have the case $R_{0} \leq 1$ and $R_{0}>1$. The parameters values used in the 
simulation are:

$$
B=100 ; \mu_{S}=0.1 ; \mu_{I}=0.02 ; \mu_{R}=0.03 ; \gamma=0.2 ; \beta=0.00021 ; c=0.2 ;
$$

from this value we have $R_{0}=0.095<1$.

When we change the value of $\beta$ by $\beta=0.021$, we get $R_{0}=9.54>1$. It' is important to notice that the software used is Scilab and the time is in term of weeks or months. In our graphic the red curve give the evolution of the class in the discrete model and the dashed ones give the evolution of the class in continuous-time model.

Figure 1 present the evolution of the susceptibles population through the time, the dashed cuve represent the discrete model and the red one the continuous model when $R_{0} \leq 1$.

Figure 2 give dynamic of the infected population along the time, the dashed cuve represent the discrete model and the red one the continuous model when $R_{0} \leq 1$.

Figure 3 show the evolution of the recovered population through the time, the dashed cuve represent the discrete model and the red one the continuous model when $R_{0} \leq 1$.

Figure 4 (Susceptibles population), Figure 5 (Infected population) and Figure 6 (Recovered population) represent the evolution through the time of the population when $R_{0}>1$. The dashed cuves represent the discrete model and the red one the continuous model.

For all these cuves, we can see the convergence of the red cuves (the discrete model) and the dashed ones (the continuous model)

\section{Conclusion}

In this paper, we have studied a discrete $S I R$ epidemic model with general

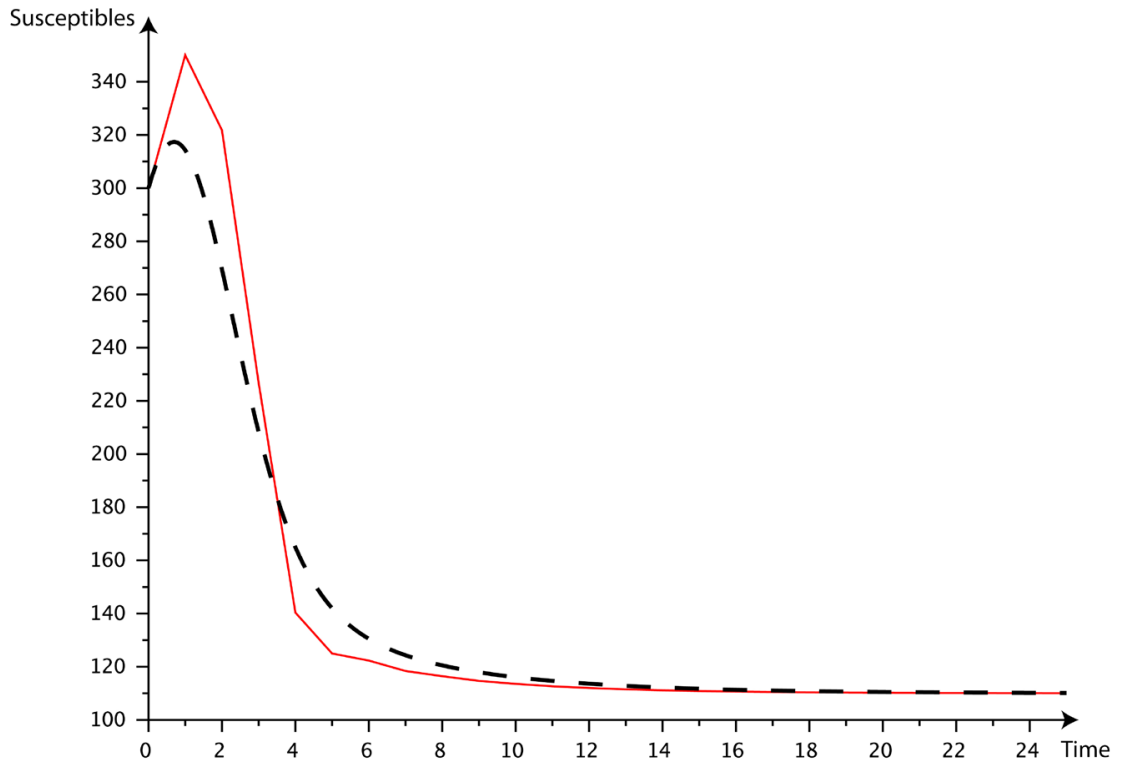

Figure 1. Graphic of susceptibles class, when $R_{0}>1$. 
Infected

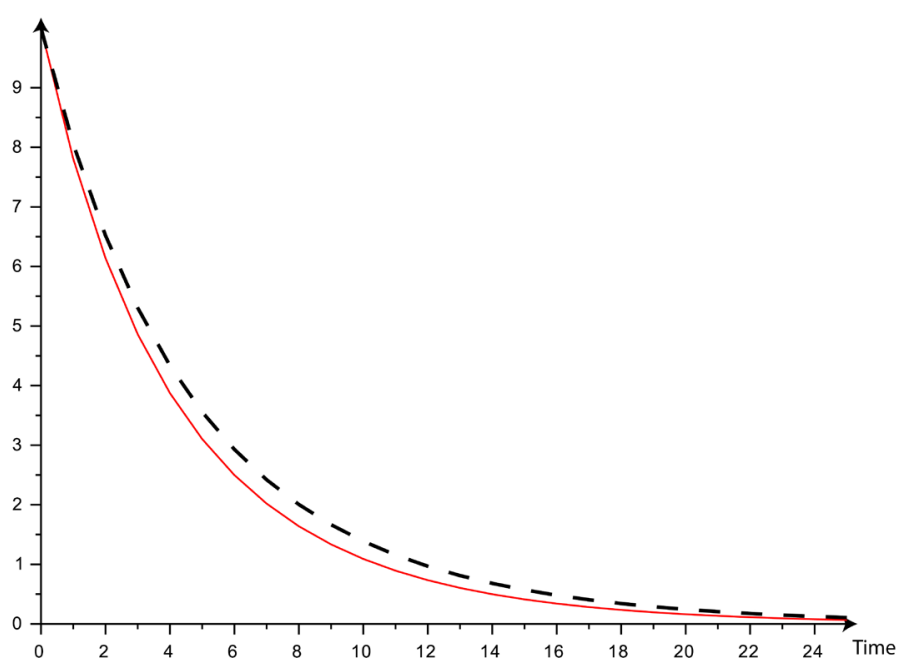

Figure 2. Graphic of infectious class, when $R_{0} \leq 1$.

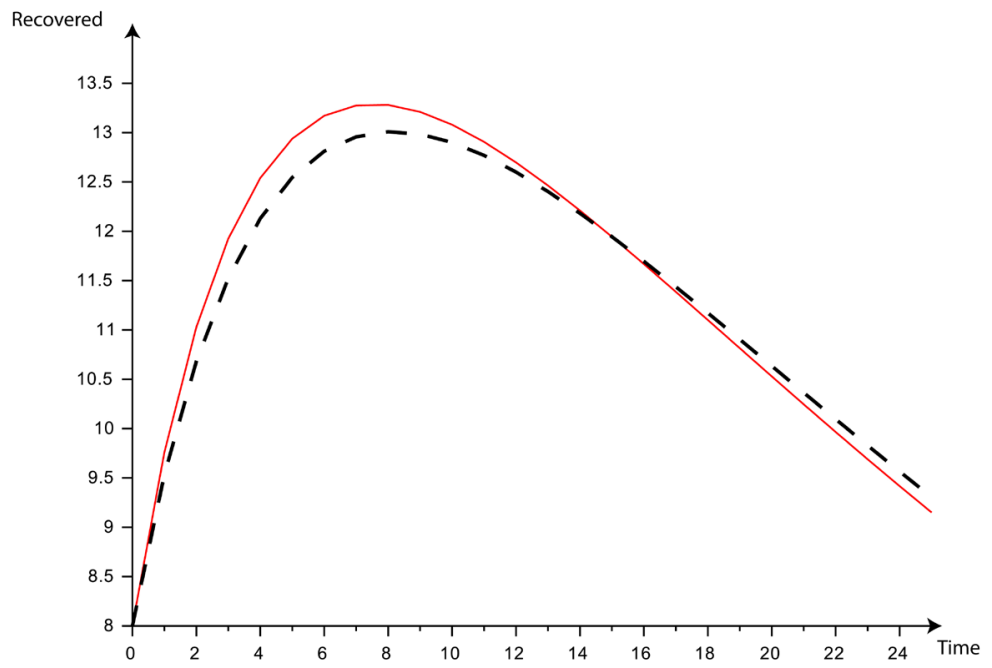

Figure 3. Graphic of recovered class, when $R_{0} \leq 1$.

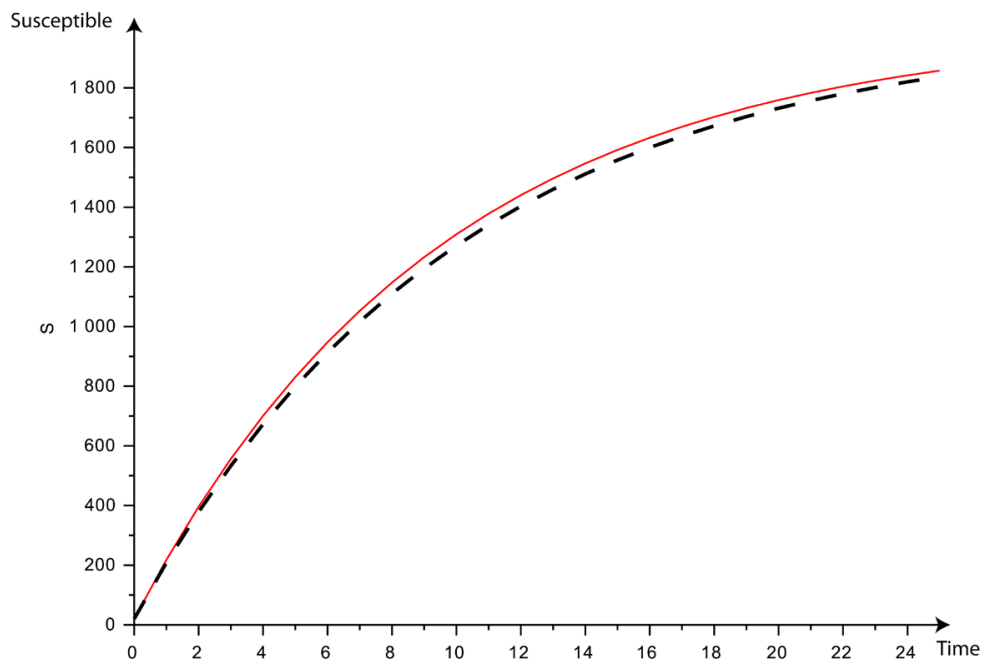

Figure 4. Graphic of susceptibles class, when $R_{0} \leq 1$. 


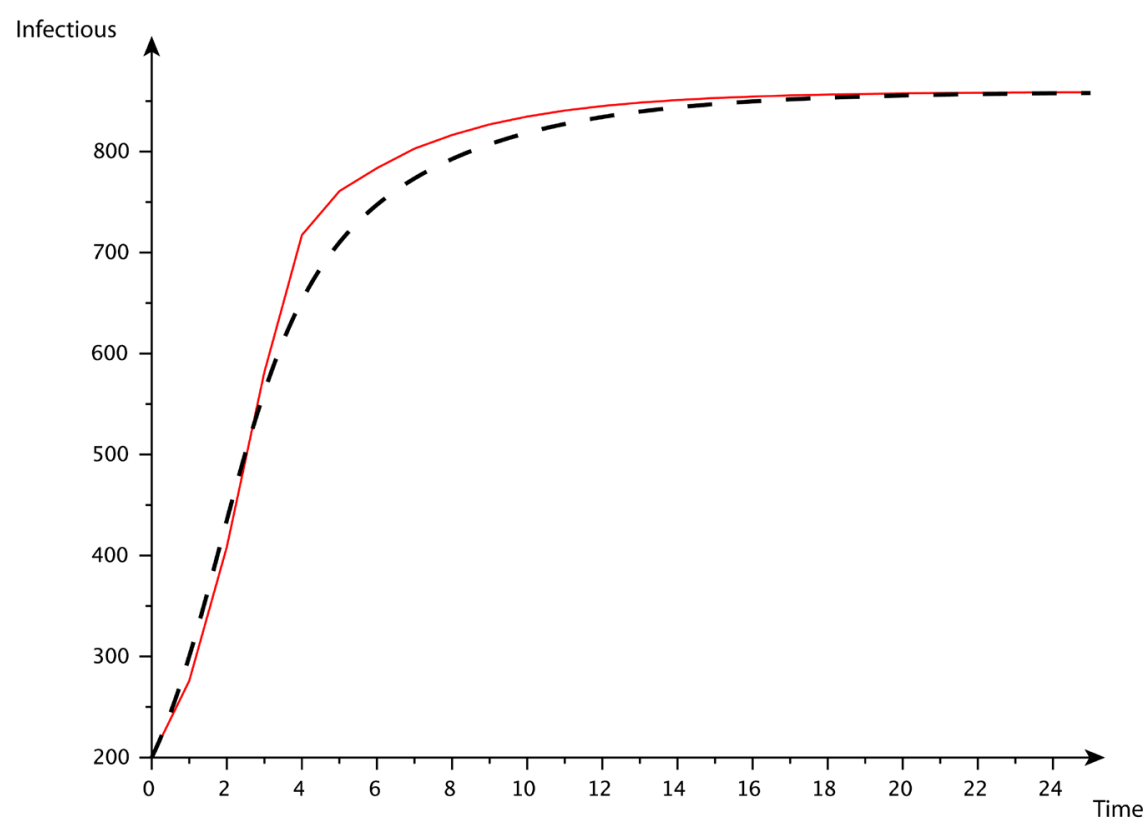

Figure 5. Graphic of infectious class, when $R_{0}>1$.

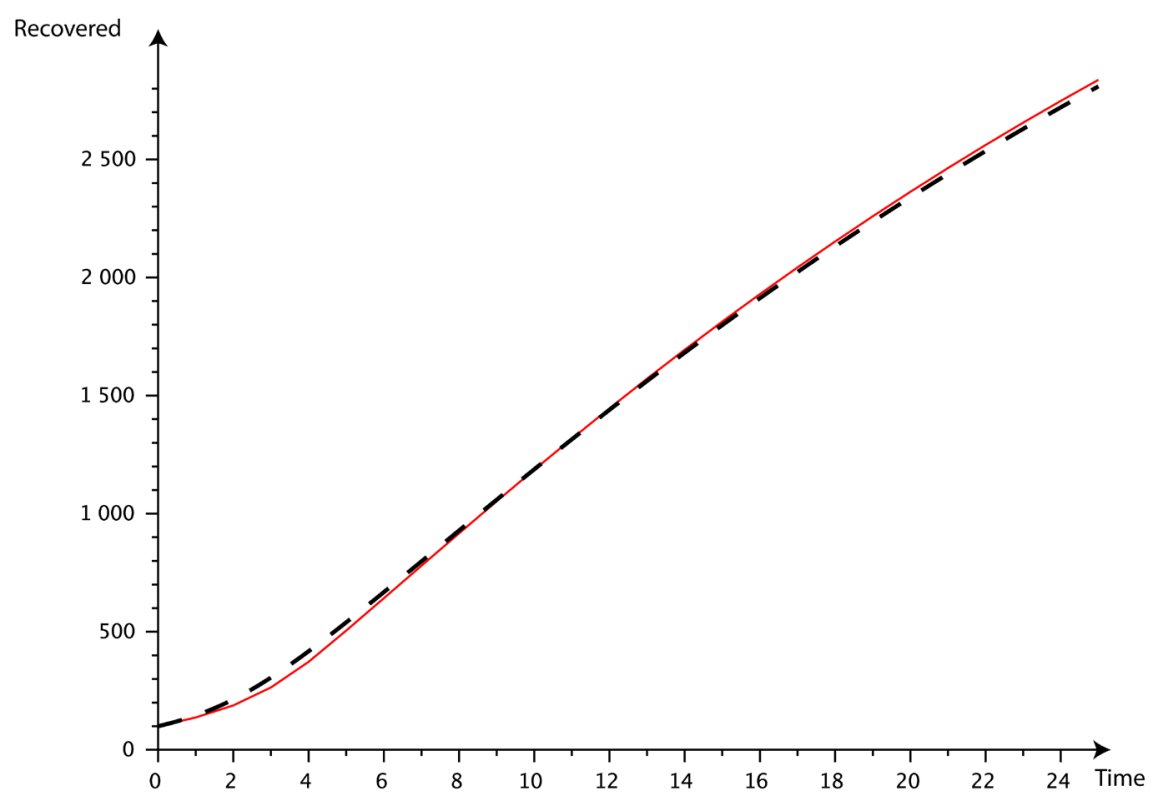

Figure 6. Graphic of recovered class, when $R_{0}>1$.

incidence. We have proved the global stability of discrete SIR epidemic model by using the comparison theorem from the global stability of disease free equilibrium, when $R_{0} \leq 1$, on the positive invariant set $K$ and we have also proved the local stability of disease free equilibrium. The technique of Lyapunov function is used to proved the global stability of endemic equilibrium, when $R_{0}>1$. We have made the numerical simulation to corroborate theoretical results. From the results obtained in this paper, we can conclude that the Euler backward difference scheme, that is, the discrete dynamical model (2), is obtained with excellent 
dynamical properties for the step size $\tau=1$ in the local and global stability of equilibra. These properties are nearly the same as the corresponding continuous-time model (1). In our future work, it shall be important for us to study the same model, but with general positive step size $\tau$ and see how bifurcation can happen.

\section{Acknowledgments}

The authors want to thank the anonymous referee for his valuable comments on the paper.

\section{Author's Contribution}

Aboudramane Guiro provide the subject, wrote the introduction and the conclusion and verified some calculation. Dramane Ouédraogo conceived the study and computed the equilibria and their local stabilities. Harouna Ouédraogo rote mathematical formula, bring up the Lyapunov functional and did all the calculus with the other authors. All the authors read and approved the final manuscript.

\section{Conflicts of Interest}

The authors declare that they have no competing interests.

\section{References}

[1] Zhang, T. and Teng, Z. (2008) Global Behavior and Permanence of SIRS Epidemic Model with Time Delay. Nonlinear Analysis. Real World Applications, 9, 1409-1424. https://doi.org/10.1016/j.nonrwa.2007.03.010

[2] Cooke, K.L. (1979) Stability Analysis for a Vector Disease Model. Rocky Mountain Journal of Mathematics, 9, 31-42. https://doi.org/10.1216/RMJ-1979-9-1-31

[3] Guiro, A., Ouédraogo, D. and Ouédraogo, H. Global Stability for a Discrete SIR Epidemic Model with Delay in the General Incidence Function. Submitted to Advances in Difference Equations.

[4] Hethcote, H.W. (1976) Qualitative Analyses of Communicable Disease Models. Mathematical Biosciences, 28, 335-356. https://doi.org/10.1016/0025-5564(76)90132-2

[5] Hethcote, H.W. (2000) The Mathematics of Infectious Diseases. SIAM Review, 42, 599-653. https://doi.org/10.1137/S0036144500371907

[6] Takeuchi, Y., Ma, W. and Beretta, E. (2000) Global Asymptotic Properties of a Delay SIR Epidemic Model with Finite Incubation Times. Nonlinear Analysis, 42, 931-947. https://doi.org/10.1016/S0362-546X(99)00138-8

[7] Beretta, E., Hara, T., Ma, W. and Takeuchi, Y. (2001) Global Asymptotic Stability of an SIR Epidemic Model with Distributed Time Delay. Nonlinear Analysis, 47, 4107-4115. https://doi.org/10.1016/S0362-546X(01)00528-4

[8] Ma, W., Takeuchi, Y., Hara, T. and Beretta, E. (2002) Permanence of an SIR Epidemic Model with Distributed Time Delays. Tohoku Mathematical Journal, 54, 581-591. https://doi.org/10.2748/tmj/1113247650

[9] Capasso, V. and Serio, G. (1978) A Generalization of the Kermack-McKendrick Deterministic Epidemic Model. Mathematical Biosciences, 42, 43-61. 
https://doi.org/10.1016/0025-5564(78)90006-8

[10] Xu, R. and Ma, Z. (2009) Global Stability of a SIR Epidemic Model with Nonlinear Incidence Rate and Time Delay. Nonlinear Analysis. Real World Applications, 10, 3175-3189. https://doi.org/10.1016/j.nonrwa.2008.10.013

[11] Connell McCluskey, C. (2010) Global Stability of an SIR Epidemic Model with Delay and General Nonlinear Incidence. Mathematical Bioscience and Engineering Volume 7, Number 4.

[12] Yoichi, E. and Yukihiko, N. (2010) Global Stability for a Class of Discrete SIR Epidemic Models. Mathematical Biosciences and Engineering, Volume 7, Number 2.

[13] Jang, S. and Elaydi, S.N. (2003) Difference Equations from Discretization of a Continuous Epidemic Model with Immigration of Infectives. Canadian Applied Mathematics Quarterly, 11, 93-105.

[14] Izzo, G. and Vecchio, A. (2007) A Discrete Time Version for Models of Population Dynamics in the Presence of an Infection. Journal of Computational and Applied Mathematics, 210, 210-221. https://doi.org/10.1016/j.cam.2006.10.065

[15] Izzo, G., Muroya, Y. and Vecchio, A. (2009) A General Discrete Time Model of Population Dynamics in the Presence of an Infection. Discrete Dynamics in Nature and Society, Art. ID: 143019, 15 p. https://doi.org/10.1155/2009/143019

[16] Sekiguchi, M. (2009) Permanence of Some Discrete Epidemic Models. International Journal of Biomathematics, 2, 443-461. https://doi.org/10.1142/S1793524509000807

[17] Hale, J. and Verduyn Lunel, S. (1993) Introduction to Functional Differential Equations, Springer-Verlag. https://doi.org/10.1007/978-1-4612-4342-7

[18] Van den Driesche, P. and Watmough, J. (2002) Reproduction Numbers and Substhreshold Endemic Equilibria for the Compartmental Model of Disease Transmission. Mathematical Biosciences, 180, 29-48.

[19] Lakshmikantham, V., Leela, S. and Martynyuk, A.A. (1989) Stability Analysis of Nonlinear Systems. Marcel Dekker, New York.

[20] Lasalle, J.P. (1976) The Stability of Dynamical Systems. SIAM, Philadelphia. https://doi.org/10.1137/1.9781611970432 